\title{
Artículos
}

\section{¿Las ciudades inteligentes ayudan a combatir el desempleo? Un análisis multinivel}

\section{Do smart cities help to confront unemployment? A multilevel analysis}

\author{
María Verónica Alderete*
}

\section{Resumen}

El cambio propiciado por las Tecnologías de la Información y Comunicación (TIC) ha conducido a examinar la relación entre las TIC y el desempleo. El objetivo de este trabajo es analizar si la mayor difusión de las TIC que caracteriza a las ciudades inteligentes explica sus mayores niveles de desempleo. Con este fin, se emplea una muestra de 138 ciudades pertenecientes a 63 países, para las cuales está publicado el indice Cities in Motion. Se estima un modelo de regresión multinivel con dos niveles: ciudad y país. Los resultados obtenidos establecen que la tecnología en ambos niveles de análisis explica significativamente las tasas de desempleo a nivel ciudad.

Palabras clave: Tecnologías de la Información y de la Comunicación (TIC); ciudades; desempleo; modelo multinivel.

\footnotetext{
Abstract

Changes due to Information and Communication Technologies (ICT) diffusion have fostered the need to examine the relationship between ICT and unemployment. The objective of this paper is analyzing whether a higher ICT diffusion, proper of smart cities, explains a higher unemployment rate. To this end, we employ a sample of 138 cities from 64 countries. These cities are those for whom the Cities in Motion Index are published. We estimate a multilevel regression model, with two levels: city and country. Results obtained show that technology at both levels of analysis significantly explains cities unemployment rates.

Keywords: Information and Communication Technologies; cities; unemployment; multilevel model.

* Universidad Nacional del Sur - Conicet, Instituto de Investigaciones Económicas y Sociales del Sur (IIESS), Departamento de Economía. Dirección postal: San Andrés 800, Altos de Palihue, Bahía Blanca, 8000, Argentina. Correo electrónico: mvalderete@iiess-conicet.gob.ar 


\section{Introducción}

El desempleo ha sido una cuestión de debate durante muchos años tanto entre académicos como políticos en el ámbito de las naciones, y aún sigue siéndolo. En el siglo pasado, varios estudios con datos a nivel países han examinado la relación entre innovación y desempleo (Freeman, Clark y Soete, 1983; Gomulka, Ostaszewski y Davies, 1990; García Sánchez, 1993; Audretsch, 1995; Pianta, 2005). Más recientemente, el cambio en la vida diaria propiciado por la adopción de las nuevas Tecnologías de la Información y de la Comunicación (TIC) ha conducido a la necesidad de examinar la relación entre las TIC y el desempleo.

Varios investigadores analizaron la dinámica del desempleo a nivel países y sus probables causas, desde la presencia de shocks de demanda agregada hasta las características institucionales. Sin embargo, es escasa la evidencia empírica respecto del desempleo en las ciudades (García y Sánchez, 2012; González Oliva, Karldotter, Martin y del Amo, 2010; Khattab, 2006; Giles, Park y Zhang, 2005) y su vinculación con el grado de difusión de las nuevas TIC, tanto a nivel ciudad como a nivel país (Acemoglu y Restrepo, 2017).

Los especialistas se refieren al siglo XXI como el siglo de las ciudades, en parte debido a dos tendencias mundiales: la urbanización y la informatización. Ambas tendencias han convergido dando lugar a la gestión inteligente para garantizar la competitividad y la sustentabilidad de las ciudades.

Este trabajo se enmarca en el concepto de Ciudad Inteligente o Smart City, del cual existen múltiples definiciones. Éste se caracteriza por el uso cotidiano de la tecnología para mejorar la eficiencia en respuesta a las demandas ciudadanas. Hollands (2008) describe la Ciudad Inteligente a partir de cuatro características: a) la aplicación de tecnologías digitales y electrónicas a las ciudades y sus residentes; $b$ ) el uso de TIC para transformar la vida y el trabajo; c) el involucramiento entre las TIC y los habitantes; y d) el mejoramiento de la innovación, aprendizaje, conocimiento y resolución de problemas usando las tecnologías.

La brecha o división entre aquellos que tienen un acceso regular y efectivo a las tecnologías digitales, en particular internet, y aquellos que no, se conoce como brecha digital (United Nations, 2006; Van Dijk y Hacker, 2006, 2003). La brecha digital puede ser categorizada como global, regional o nacional (Rao, 2005). A nivel nacional existe una brecha en el interior de los países que distingue entre las regiones o áreas urbanas y rurales. Por tal motivo, focalizar el análisis en ciudades capitales o metropolitanas permite controlar por las disparidades entre lo urbano y lo rural en términos de de- 
sarrollo humano y progreso económico, especialmente en los países en desarrollo (Furuholt y Kristiansen, 2007; Mwesige, 2004), y las diferencias en cuanto a las habilidades digitales y culturales (Van Dijk, 2006). Las capacidades y habilidades para usar las computadoras han sido influenciadas por las oportunidades para desarrollar y acumular el capital cultural (Clayton y Macdonald, 2013). En este sentido, es probable que las ciudades capaces de aprovechar las ventajas de las TIC sean aquellas que provean un capital cultural acorde a las necesidades.

Tal como indica Barrón (2013), el análisis del desempleo tiene que hacerse separando a la población urbana de la rural, pues los comportamientos son diferenciados ya que la posibilidad de conseguir empleo en las áreas urbanas es mayor que en las rurales. Esta situación se evidencia más aún ante la difusión de las nuevas TIC en la sociedad. Es menos probable que la población rural use las nuevas tecnologías como consecuencia de los mayores costos de infraestructura. A su vez, es menor la probabilidad de usar computadoras o internet en el trabajo en áreas rurales (Vicente y López, 2006).

Las actividades relacionadas con el sector de las TIC se han convertido en un motor económico clave, pero también en una de las causas de los problemas sociales, incluidos el desempleo y los bajos ingresos (Clayton, Macdonald y Wilcok, 2010). Con el advenimiento de las TIC, los empleos son más fáciles de ser buscados y encontrados para las personas que están incluidas digitalmente (Mc Quaid, Lindsay y Greig, 2004). Por otro lado, mientras que algunos trabajos están destinados a desaparecer frente a la tecnología, como los trabajos físicos, como el de sembrador, o intelectuales, como el buscador de datos, otros nuevos trabajos emergieron. Por ejemplo, los instructores físicos son cada vez más solicitados ante la vida más sedentaria de la población.

Sin embargo, no hay suficiente evidencia empírica de que exista una relación positiva entre los TIC y el desempleo. En algunos países se han registrado incrementos en la tasa de desempleo durante los últimos años ante las reducciones en las inversiones en TIC (Japón), mientras que aquellos países que invirtieron en TIC lograron reducir la tasa de desempleo (Italia, España) (Rillo, 2015). Por otro lado, en ciudades como Bruselas — sin ser percibida como una ciudad inteligente y con un puntaje bajo, según algunos índices de Smart City-, se enfrentan altas tasas de desempleo de aproximadamente $19.3 \%$, mucho más altas que el 5.1\% de desempleo en la región de Flandes (Walravens, 2015). De forma similar ocurre en Nairobi, con altas tasas de desempleo y bajos niveles tecnológicos; sin embargo, existen actualmente esfuerzos de su gobierno para modificar esta situación a partir del desarrollo del complejo Ciudad Tecnológica, que se inaugurará en 2025. Este 
complejo ofrecerá vivienda y trabajo para aproximadamente 200 mil profesionales.

Por otra parte, se observa que existen grandes diferencias entre las tasas de desempleo a nivel nacional y el desempleo a nivel de las ciudades. En Brasil, las ciudades de Río de Janeiro y Brasilia poseen una tasa de desempleo (18.9 y $18.1 \%$ respectivamente) superior a la media nacional, de $12 \%$, según datos de 2016 (knoema.es). La misma situación se observa en Cali (Colombia), Lima (Perú) y Guadalajara (México), con niveles de desempleo por encima del promedio nacional, entre otros casos. Asimismo, en Letonia, aunque la tasa de desempleo se redujo de $17.3 \%$ en marzo de 2010 a $7.9 \%$ en septiembre de 2016, se observan diferencias entre las regiones. La región de Riga posee la menor tasa de desempleo (5.1\%), tres veces más baja que la región de Latgale, al este de Letonia, que padece la tasa de desempleo más alta (17.4\%).

Debido a que en los últimos años tanto la investigación como la política han puesto el foco en el desarrollo de las ciudades, este trabajo tiene como objetivo examinar la relación entre las TIC y el desempleo en las urbes. ¿Las ciudades inteligentes o más tecnológicas tienen menores tasas de desempleo que el resto de las ciudades? Con vistas a dar respuesta a este interrogante, se utiliza una muestra de 138 ciudades pertenecientes a 63 países. Las urbes seleccionadas fueron aquellas para las cuales está publicado el índice Cities in Motion de la Escuela de Negocios IESE, de la Universidad de Navarra. Se estima un modelo de regresión multinivel con dos niveles: el primero corresponde a las ciudades y el segundo a los países. El trabajo se estructura de la siguiente manera. En primer lugar, se describe el marco teórico referido al concepto de Ciudad Inteligente, y a los cambios registrados en el mercado laboral frente al advenimiento tecnológico. Posteriormente, se analiza la metodología de Modelos Multinivel enmarcada en los objetivos del trabajo. A continuación, se estima el modelo y se discuten los resultados. Finalmente, se presentan las conclusiones e implicancias del trabajo.

\section{Marco teórico}

La Ciudad Digital o Ciudad con Inteligencia se caracteriza por el uso cotidiano de la tecnología para mejorar la eficiencia en respuesta a las demandas ciudadanas. Por otro lado, se encuentra el concepto de Ciudad Inteligente, basado en un enfoque integral que debe incluir otros aspectos más allá de los tecnológicos, como la preocupación medioambiental y el desarrollo en la calidad de vida de los ciudadanos como fin último. Por lo tanto, Ciudad 
con Inteligencia no es lo mismo que Ciudad Inteligente, la cual refiere a un enfoque mucho más holístico e integrador, en donde la tecnología es un factor necesario pero no suficiente para resolver problemas, mejorar la eficiencia y desarrollar la calidad de vida de los ciudadanos (Jolías y Prince, 2016).

La literatura de Ciudad Inteligente generalmente define las características sociales, económicas, tecnológicas y ambientales que convierten a una ciudad en más "inteligente" que otra. Para algunos, el concepto responde al rol de la tecnología para enlazar a los habitantes con las instituciones (Belissent y Girón, 2013) o en su posición relativa dentro de las redes mundiales (Wall y van der Knaap, 2011); para otros, en la importancia del capital humano para reducir la brecha entre educación y productividad (Florida y Mellander, 2012).

La noción de Ciudad Inteligente ha evolucionado desde la ejecución de proyectos específicos hasta la instrumentación de estrategias globales para enfrentar los desafíos de las urbes más grandes. La implementación del concepto implica el compromiso de múltiples agentes, sector público, sector privado, academia y ciudadanos para impulsar tanto el capital físico como el intelectual y el social (Dameri, 2014).

Cualquier Ciudad Inteligente debe dar respuesta a los obstáculos y oportunidades que conviven en las ciudades del siglo XXI, tales como el desempleo. En la medida que los robots y las tecnologías asistidas por las computadoras realizan tareas anteriormente desempeñadas por el trabajo, existe una preocupación creciente por el futuro del empleo (Acemoglu y Restrepo, 2017). Estos autores analizan el efecto del uso de los robots en el mercado laboral de Estados Unidos, y encuentran que si los robots compiten con el trabajo humano, éstos pueden reducir el empleo. A su vez, algunos economistas han examinado la frecuencia de búsquedas de trabajo en Google para comprender los cambios en el empleo (Wu y Brynjolfsson, 2013).

Durante los siglos XIX y XX, el empleo frecuentemente se reestablecía luego de cada recesión; pero a partir de 1990 el empleo no se recuperó como antes. No es coincidencia que la informatización e ingreso de las computadoras en la economía hayan avanzado en esa época y que los patrones de contratación hayan cambiado luego de las recesiones (Brynjolfsson y McAfee, 2015).

Existe cierta evidencia de que la automatización de un grupo de ocupaciones o trabajos de baja y media calificación ha contribuido a la polarización en el empleo (Michaels, Nastraj y Van Reenen, 2014; Goos y Maning, 2007; Autor y Dorn, 2013), así como al desempleo en los trabajos más rutinarios. Los trabajos rutinarios donde la tecnología es un sustituto han disminuido a 
$11 \%$ entre 2001 y 2011 (Jaimovich y Siu, 2012). Mientras que algunos trabajos, generalmente basados en mano de obra no calificada, son sustituidos por las TIC (los que escriben a máquina, las secretarias), otros trabajos calificados surgen como un complemento de las TIC (como asistencia de gerencia). Luego, existe una correlación entre la innovación tecnológica y el empleo (Castells y Cardoso, 2005; Parkin, 2007).

Tal como expresan Acemoglu y Restrepo (2017), a pesar de estas evidencias, el impacto de las nuevas tecnologías en el mercado laboral depende no sólo de en dónde (qué sector) afectan directamente, sino también en el ajuste de otras partes de la economía. El impacto negativo de los robots en el empleo se explica debido a un efecto desplazamiento (al reemplazar a los trabajadores en las tareas). Sin embargo, las nuevas tecnologías también ejercen un efecto positivo debido al efecto productividad (en la medida que otras industrias y/o tareas aumentan su demanda de trabajo).

Acemoglu y Restrepo (2017) explican el impacto sobre el equilibrio de un solo tipo de tecnología: los robots industriales en el mercado laboral americano. Los autores se refieren a tales robots, pero excluyen otros tipos de capital, tales como el software u otras tecnologías. Por otro lado, su objeto de estudio son los sectores productivos o industrias, más que las ciudades propiamente dichas. Por lo tanto, los resultados del presente trabajo podrían complementar los recientemente realizados sobre los robots, al incluir las nuevas TIC teniendo en cuenta el rol que las mismas ejercen en las ciudades metropolitanas o grandes urbes donde predomina el sector servicios (Trejo Nieto, 2017; Rubiales Pérez, 2016).

Varios autores han demostrado la importancia significativa de las inversiones en TIC en la productividad de las empresas de servicios (Nath y Liu, 2017; Alderete y Gutiérrez, 2012; Sapprasert, 2010). Tanto los avances observados en las TIC, que permiten que una mayor cantidad de información sea codificada y transferida, como la tendencia creciente de difusión de las tecnologías del conocimiento, han expandido el alcance del uso de las TIC en varios servicios (Maroto Sánchez, 2012; Pilat, 2001; Licht y Moch, 1999). Porter (1990) enumera e identifica la importancia del rol de las ciudades y las regiones, destacando que las bases para las ventajas competitivas son intensamente locales, incluyendo el proceso de creación de skills, como la calidad y cantidad de capital humano, infraestructura y tecnología, los cuales varían entre regiones. Este proceso implica que los ciudadanos podrán recibir servicios urbanos optimizados a través de las TIC, siempre que sean capaces de acceder a las tecnologías y desarrollar las habilidades digitales necesarias. Aquellos ciudadanos que reúnan las habilidades apropiadas podrán crecientemente crear oportunidades y elegir cómo, dónde y cuándo trabajar. 
Por el contrario, aquellos sin estas habilidades no podrán en el futuro mejorar su situación. Esto conduce a una polarización de la población. Frente a esta situación, es necesario invertir en las capacidades de los empleados para aumentar la resiliencia de la población y de las organizaciones, así como redefinir las ya existentes. Las capacidades y habilidades siempre fueron necesarias, pero actualmente el ciclo de vida de las mismas es muy corto (Manpower Group, 2017).

Clayton y Macdonald (2013) estudian el rol que las TIC juegan en la ciudad de Sunderland, considerada una de las ciudades más conectadas digitalmente de Inglaterra. La tecnología es más significativa para el sector de clase media que para los sectores socialmente excluidos, debido a que pueden acceder a las tecnologías emergentes y a la capacitación por medio de su lugar de trabajo. Mientras que para los trabajadores calificados la tecnología ha influido fuertemente en su empleo y en sus perspectivas laborales, en el caso de los trabajadores no calificados las TIC no han tenido ningún impacto. A pesar de este resultado, la mayoría de los encuestados siente que la tecnología incrementa las oportunidades de empleo en el área local.

Pearson (2006) explora el rol de las TIC en crear ciudades que sean lugares prósperos para vivir y trabajar. Si los robots gradualmente automatizan los trabajos físicos, y las computadoras automatizan gradualmente el trabajo intelectual, luego los que restan son aquellos trabajos basados en capacidades humanas núcleo, habilidades interpersonales, contacto personal, servicios personales, seguridad, enseñanza, liderazgo y habilidades motivacionales (Pearson, 2006, p.12). La gobernanza urbana debería ser responsable de crear los trabajos y de mejorar el bienestar (Tolga Akcura y Burcu Avci, 2014).

Por último, siguiendo la literatura internacional, varios autores han incluido el tamaño de la población o la densidad demográfica (Bernal-Verdugo, Furceri y Giuillaume, 2012; Crivelli, Furceru y Toujas-Bernate, 2012) para capturar condiciones demográficas de los países, tales como su tamaño. Los países más grandes disponen de una mayor infraestructura y efectos de sinergia que pueden conducir a menores niveles de desempleo (Taylor y Bradley, 1997).

\section{Metodología}

Este trabajo se basa en una estructura de datos multinivel jerárquica, ya que por un lado se elige una estructura de datos mayor (países) y luego se seleccionan casos dentro de la misma (ciudades). Por lo tanto, se selecciona una 
muestra por etapas donde existe dependencia de las observaciones dentro de cada grupo (Hox, 1995).

Resulta conveniente utilizar este tipo de estructura multinivel para analizar el impacto de las nuevas tecnologías sobre la tasa de desempleo de las ciudades, teniendo en cuenta la heterogeneidad del desempleo entre las urbes de un mismo país, así como la heterogeneidad entre los países. De esta manera, resulta más precisa la distinción de los efectos de las TIC generados por cada uno de esos niveles. Existen algunos trabajos que han utilizado este tipo de estructura para el análisis del desempleo (García y Sánchez, 2012; González Oliva, Karlsdotter, Martín y Del Amo González, 2010; Khattab, 2006; Giles, Park y Zhang, 2005).

En el presente trabajo, la muestra se compone de 138 ciudades del mundo agrupadas en 64 países. El número promedio de ciudades por país es de dos.

Las urbes incluidas en la muestra fueron aquellas para las cuales se disponía de información sobre tasa de desempleo, así como indicadores de desarrollo tecnológico o indicadores TIC. Con respecto a estos últimos, se utilizó la información proveniente del índice Cities in Motion (CIMI).

El índice CIMI es publicado por el IESE, Escuela de Negocios del Departamento de Estrategia de la Universidad de Navarra, en conjunto con el Centro para la Globalización y la Estrategia. Este trabajo emplea como variable explicativa de TIC el ranking de Tecnología del CIMI, el cual evalúa 181 diferentes ciudades en relación con diez dimensiones: economía, capital humano, tecnología, medio ambiente, gobernanza, entre otras. Tecnología es un subcomponente del índice. ${ }^{1}$ El desarrollo tecnológico es una dimensión que permite a las ciudades ser sustentables a lo largo del tiempo y mantener o extender las ventajas competitivas de su sistema productivo y de la calidad del empleo. Este subcomponente incluye nueve indicadores, entre los cuales figuran el número de suscripciones de banda ancha en el país, la cantidad de usuarios de banda ancha en la ciudad, el número de direcciones de IP per cápita, el número de usuarios de Facebook per cápita, el número de teléfonos móviles per cápita, entre otros.

El IESE publica los datos del ranking de las ciudades en este subcomponente. Éstas son ubicadas en el ranking de forma tal que las más tecnológicas ocupan los primeros puestos (siendo 1 el primer puesto) y las menos tecnológicas los puestos más bajos (siendo 181 el último). Los países con una mayor cantidad de ciudades representadas según este índice son Estados

${ }^{1}$ Según el IESE, la ponderación atribuida a Tecnología en la construcción del índice CIMI es de 0.3763 . 
Unidos (12), seguido de China y Brasil, ambos con 9 urbes. Por otro lado, hay países con información sobre una sola ciudad, tales como Noruega o Perú.

La muestra finalmente se conformó con las siguientes 138 ciudades (véase el Anexo). Cabe destacar que hay países que no fueron incluidos en la muestra al no existir datos sobre tasa de desempleo para ninguna de sus ciudades. Como se puede observar en el Anexo, en general los países con mayor población, tales como China y Brasil, poseen un mayor número de ciudades incluidas en la muestra.

La muestra incluye tanto países desarrollados y en desarrollo, como países de diferentes continentes del mundo. Con respecto a las ciudades, en general la información disponible se refiere a las metropolitanas, quedando excluidas las periféricas o no metropolitanas.

Con estos datos se estima un modelo de regresión multinivel. La ventaja de estos modelos es que las observaciones individuales se agrupan en unidades más grandes, de manera tal que se incorpora información extra vinculada al anidamiento de datos (Diez Roux, 2002; OCDE, 2003). De esta manera se obtienen mejores estimadores de los coeficientes de regresión y de su variación (De la Cruz, 2008). Los modelos multinivel pueden elaborarse de forma simple o compleja. En el primer caso, se supone que las discrepancias entre los grupos (países) se observan en la constante o intercepto. Por lo tanto, los efectos de cada variable explicativa sobre la variable dependiente (tasa de desempleo de las ciudades) son fijos entre países. Las líneas de regresión de cada país son paralelas y sólo difieren en la ordenada al origen. Estos modelos se conocen como modelos con efectos fijos.

En el segundo caso, se introducen diferencias entre países tanto en el intercepto como en los coeficientes de algunas variables explicativas. Gráficamente, las rectas de regresión difieren en la ordenada, pero también en la pendiente. Estos modelos se conocen como modelos con efectos aleatorios.

Por lo tanto, las variables explicativas de la tasa de desempleo son agrupadas en dos niveles de análisis: ciudad (nivel 1) y país (nivel 2). La idea es no tratar a los distintos contextos (países, ciudades) como entidades homogéneas con una única y común influencia sobre la tasa de desempleo. Las variables de nivel país son iguales para todas las ciudades de la misma nación, mientras que las de nivel 1 varían de acuerdo con las características propias de cada urbe.

Los modelos multinivel intentan reproducir la complejidad del fenómeno y brindan una solución estadística para examinar en forma simultánea la influencia del entorno y de las diferencias individuales (Gaviria y Castro, 2005). Por otro lado, tienen en cuenta la diferenciación de la varianza que producen los distintos niveles de agregación. Si los efectos aleatorios resul- 
tan ser estadísticamente significativos, quiere decir que hay discrepancias en lo que los países hacen en relación con las diferencias de origen de las ciudades.

Se supone que hay un modelo de tasa de desempleo en las urbes conformado por dos niveles y que incorpora cinco variables explicativas, tres de nivel ciudad y dos de nivel país.

Nivel 1:

$$
\mathrm{Y}_{\mathrm{ij}}=\beta_{0 \mathrm{j}}+\beta_{1 \mathrm{j}} \mathrm{X}_{\mathrm{ij}}+\beta_{2 \mathrm{j}} \mathrm{Z}_{\mathrm{ij}}+\mathrm{r}_{\mathrm{ij}}
$$

Nivel 2:

$$
\begin{aligned}
& \beta_{0 \mathrm{j}}=\gamma_{00}+\gamma_{01} \mathrm{P}_{\mathrm{j}}+\mu_{0 \mathrm{j}} \\
& \beta_{1 \mathrm{j}}=\gamma_{10} \\
& \beta_{2 \mathrm{j}}=\gamma_{20}+\mu_{2 \mathrm{j}}
\end{aligned}
$$

Modelo completo:

$$
Y_{i j}=\gamma_{00}+\gamma_{01} P_{j}+\gamma_{10} X_{i j}+\gamma_{20} Z_{i j}+\mu_{0 j}+\mu_{2 j} Z_{i j}+r_{i j}
$$

Tal que:

$\mathrm{Y}_{\mathrm{ij}} \quad$ es la tasa de desempleo (esperado) de la ciudad "i”" en el país “j”".

$\beta_{0 j}$ es el intercepto de la recta de regresión para el país “ $\mathrm{j}$ ”.

$\beta_{1 j}$ es el coeficiente que acompaña a la variable explicativa $X$, incorporada al modelo con efectos fijos. Por lo tanto, $\beta_{1 j}$ es igual para todos los países y se representa mediante $\gamma_{10}$.

$\beta_{2 j}$ es el coeficiente que acompaña a la variable explicativa $Z$, incorporada al modelo con efectos aleatorios. Por lo tanto, varía entre países. Está formada por una parte fija $\left(\gamma_{20}\right)$ y una parte aleatoria $\left(\mu_{2 j}\right)$. Esta última representa la distancia residual desde el coeficiente de regresión de los países hasta $\gamma_{20}$.

$\gamma_{01}$ es el coeficiente que acompaña a la variable explicativa $P$ (variable del nivel país, por eso el subíndice es “01”). Dado que pertenece al nivel 2 , existe un valor de P por cada país "j”. Esta variable influye en el valor del intercepto $\beta_{0 \mathrm{j}}$. Si hubiera otra variable explicativa de nivel país se agregaría el término $\gamma_{02} \mathrm{P}_{\mathrm{j}}$

$r_{i j}$ es la varianza residual dentro de cada país.

$\mu_{0 \mathrm{j}} \quad$ es la varianza residual entre países. 
En primer lugar, es útil estimar un modelo sin incluir las variables explicativas, llamado Modelo Nulo. Este modelo indica qué proporción de la desigualdad en las tasas de desempleo de las ciudades se debe a diferencias entre países y qué proporción se relaciona con diferencias en el interior de los mismos. El modelo formalmente se expresa como:

$$
\mathrm{Y}_{\mathrm{ij}}=\gamma_{00}+\mu_{0 \mathrm{j}}+\mathrm{r}_{\mathrm{ij}}
$$

donde:

$\gamma_{00}$ representa los efectos fijos o determinísticos (intercepto global). $\mu_{0 \mathrm{j}} \mathrm{y}_{\mathrm{ij}}$ representan los efectos aleatorios o estocásticos.

En este caso, los $\beta_{0 \mathrm{j}}$ son iguales a las medias de los países o muy cercanos a éstas. Como puede observarse, $\beta_{0 \mathrm{j}}$ está formada por una parte fija, común a todos los países $\left(\gamma_{00}\right)$, llamado intercepto global, y una parte aleatoria $\left(\mu_{0 \mathrm{j}}\right)$, que representa la desviación del país “j” respecto de $\gamma_{00}$, es decir, representa la varianza entre países.

Por otro lado, $\mathrm{r}_{\mathrm{ij}}$ es el desvío de la tasas de desempleo de la ciudad "i" respecto del promedio del país " $\mathrm{j}$ " al que pertenece. Dado que en este modelo a cada ciudad se le asigna el promedio de su país como resultado predicho, $\mathrm{r}_{\mathrm{ij}}$ es igual a la varianza dentro de cada país.

Por lo tanto, en el modelo nulo con las varianzas residuales intrapaíses e interpaíses es posible calcular el coeficiente de correlación intraclase $(\rho)$, el cual representa la proporción de la varianza residual explicada por diferencias entre países $\left(\rho=\mu_{0 j} /\left(\mu_{0 j}+r_{i j}\right)\right.$; es decir, la proporción de la varianza de las tasas de desempleo de las ciudades entre países. Si este coeficiente fuera cero, no tendría sentido plantear un modelo multinivel (OCDE, 2003).

La bondad de ajuste de los modelos surge del indicador denominado "varianza explicada", el cual se obtiene de comparar el modelo propuesto con el modelo nulo. La manera de calcular este indicador se muestra en el Cuadro 1.

El trabajo emplea el software STATA.14 para calcular los parámetros de las ecuaciones anteriores, los cuales se estiman simultáneamente mediante métodos iterativos, cuyo objetivo es maximizar una función de máxima verosimilitud. 


\section{Cuadro 1}

Cálculo de la varianza explicada

\begin{tabular}{ll}
\hline Total & $1-\left(\mu_{0 \mathrm{j}+} \mathrm{r}_{\mathrm{ij}}\right)_{\text {Modelo propuesto }} /\left(\mu_{0 \mathrm{j}+} \mathrm{r}_{\mathrm{ij}}\right)_{\text {Modelo nulo }}$ \\
Nivel 1 (ciudades) & $1-\left(\mathrm{r}_{\mathrm{ij}}\right)_{\text {Modelo propuesto }} /\left(\mathrm{r}_{\mathrm{ij}}\right)_{\text {Modelo nulo }}$ \\
Nivel 2 (países) & $1-\left(\mu_{0 \mathrm{j}}\right)_{\text {Modelo propuesto }} /\left(\mu_{0 \mathrm{j}}\right)_{\text {Modelo nulo }}$ \\
\hline
\end{tabular}

Fuente: Elaboración propia con base en OCDE, 2003.

\subsection{Variables $y$ datos}

En esta sección se describen las variables utilizadas para estimar los modelos de regresión multinivel. Teniendo en cuenta que el objetivo del trabajo consiste en determinar el impacto de las nuevas tecnologías en la tasa de desempleo de las ciudades, las principales variables explicativas del modelo son Tecnología, seguida del Índice IDI. En el Cuadro 2 se listan y describen las variables del modelo, así como las fuentes de información consultadas.

De acuerdo con la revisión de la literatura, se espera que ambas tengan un efecto significativo y negativo sobre la tasa de desempleo. El modelo también incluye variables de control de Nivel 1 (ciudad), tales como Población y Economía; así como de Nivel 2 (país), tales como Desempleo e Industria. A continuación, se presentan las estadísticas descriptivas de las variables incluidas en el modelo (Cuadro 3):

La variable dependiente, tasa de desempleo a nivel ciudad, es una variable continua que alcanza un mínimo de 0.4 y un máximo de $43 \%$. Como puede verse en el Cuadro 3, los niveles máximos a nivel ciudad son superiores a las tasas de desempleo a nivel país.

\section{Resultados}

Para dar respuesta al interrogante del trabajo, se estima el modelo de regresión multinivel. En primer lugar, se presentan los resultados obtenidos de la estimación del modelo nulo para determinar la utilidad de un modelo multinivel. Con el modelo nulo, se busca a priori una apreciación de las variaciones en la tasa de desempleo urbano existente entre los distintos niveles, es decir, dentro de las ciudades (nivel 1) y entre las ciudades (nivel 2).

Por un lado, se analiza la significatividad de los efectos aleatorios de la constante; por otro lado, se observa el coeficiente de correlación intraclase. Como se aprecia en el Cuadro 4, los efectos aleatorios de la varianza de la 


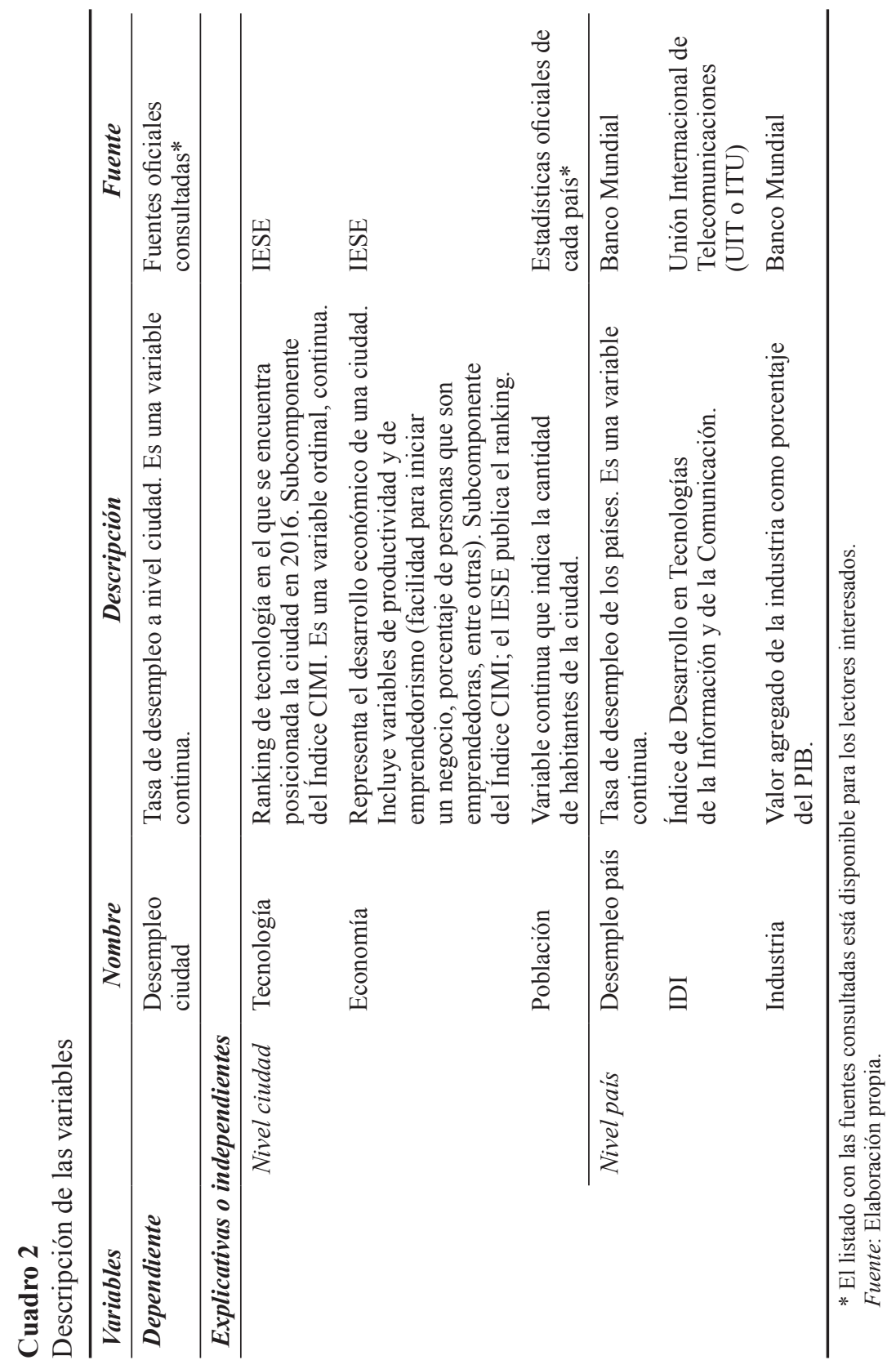




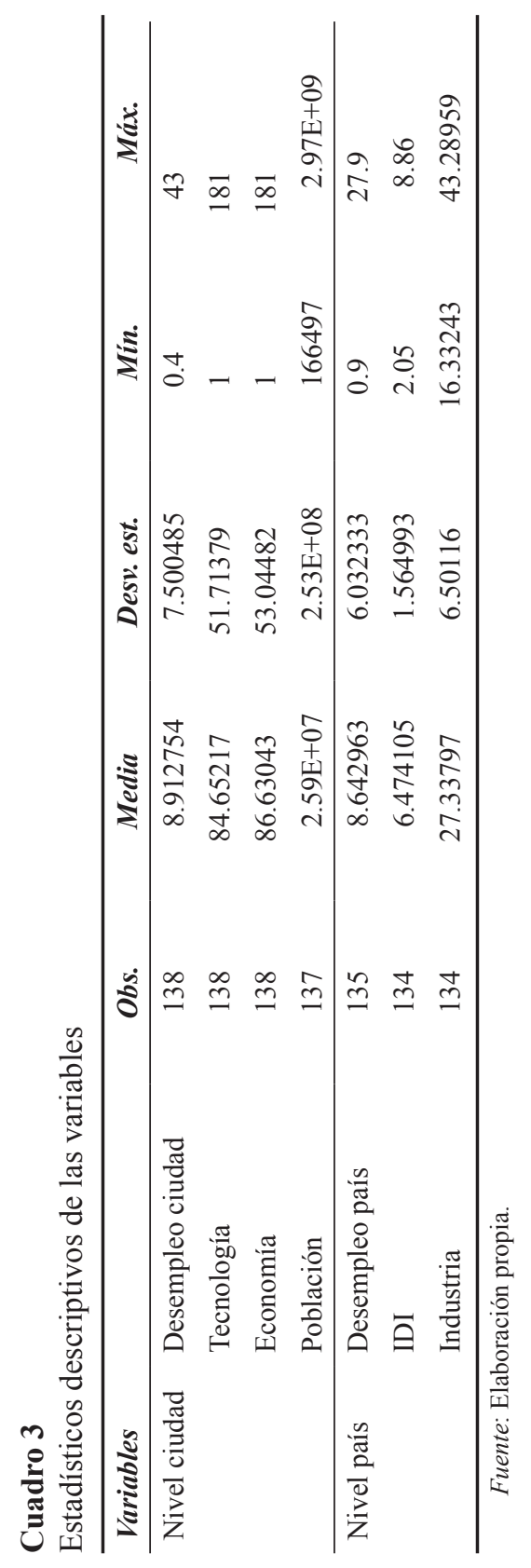




\section{Cuadro 4}

Resultados del modelo nulo

\begin{tabular}{lcc}
\hline Variables efectos fijos & Estimación & Error estándar \\
\hline Constante & 9.400427 & $1.068396^{* * *}$ \\
Efectos aleatorios de los parámetros & & \\
$\quad$ Varianza de la constante & 63.68732 & $13.3673^{* *}$ \\
$\quad$ Varianza residual & 11.72008 & $1.964991^{* *}$ \\
Coeficiente de correlación intraclase & $63.68 / 11.72=5.43$ & \\
\hline LR test vs. linear model: chibar2 $(01)=69.55$ & Prob. $>=$ chibar2 $=0.0000 \quad \mathrm{~N}=138$ \\
\hline
\end{tabular}

Nota: Estadísticamente significativo al $* 10 \%$; al $* * 5 \%$; y al $* * * 1 \%$.

Fuente: Elaboración propia.

constante son estadísticamente significativos, y la proporción de la brecha en la tasa de desempleo de las ciudades, debida a las diferencias entre países, es positiva.

Luego, se estima un modelo donde se incluyen las variables explicativas de nivel ciudad (Modelo 1). Como se destaca en el Cuadro 5, la variable Tecnología tiene signo negativo; es decir, cuanto más tecnológica es la ciudad (mejor posicionada en el ranking), mayor es la tasa de desempleo. Las ciudades ubicadas en primer lugar en el ranking de tecnología (ciudades más tecnológicas), presentan una mayor tasa de desempleo.

A continuación, para examinar si los países se comportan de manera diferente frente a las discrepancias de origen de las ciudades ante los efectos de la tecnología, se estima un modelo que incorpora efectos aleatorios a la variable Tecnología (Modelo 2, Cuadro 6).

Como puede observarse en el Cuadro 6, la variable Tecnología deja de ser estadísticamente significativa, aunque sus efectos aleatorios sí lo son. Esto significa que los países difieren en su accionar (por ejemplo, en términos de política de empleo) frente a la diferencia tecnológica de las ciudades, aunque no existe evidencia de que las ciudades puedan aplicar estrategias para modificar su situación.

Por último, se estudia un modelo (Modelo 3) que incorpora a las variables de nivel ciudad con efectos fijos, variables explicativas en el nivel país (Cuadro 7). En este caso interesa especialmente la variable IDI o nivel de desarrollo en TIC de los países, así como el desempleo y la participación de la industria en el PIB de cada uno de ellos. 


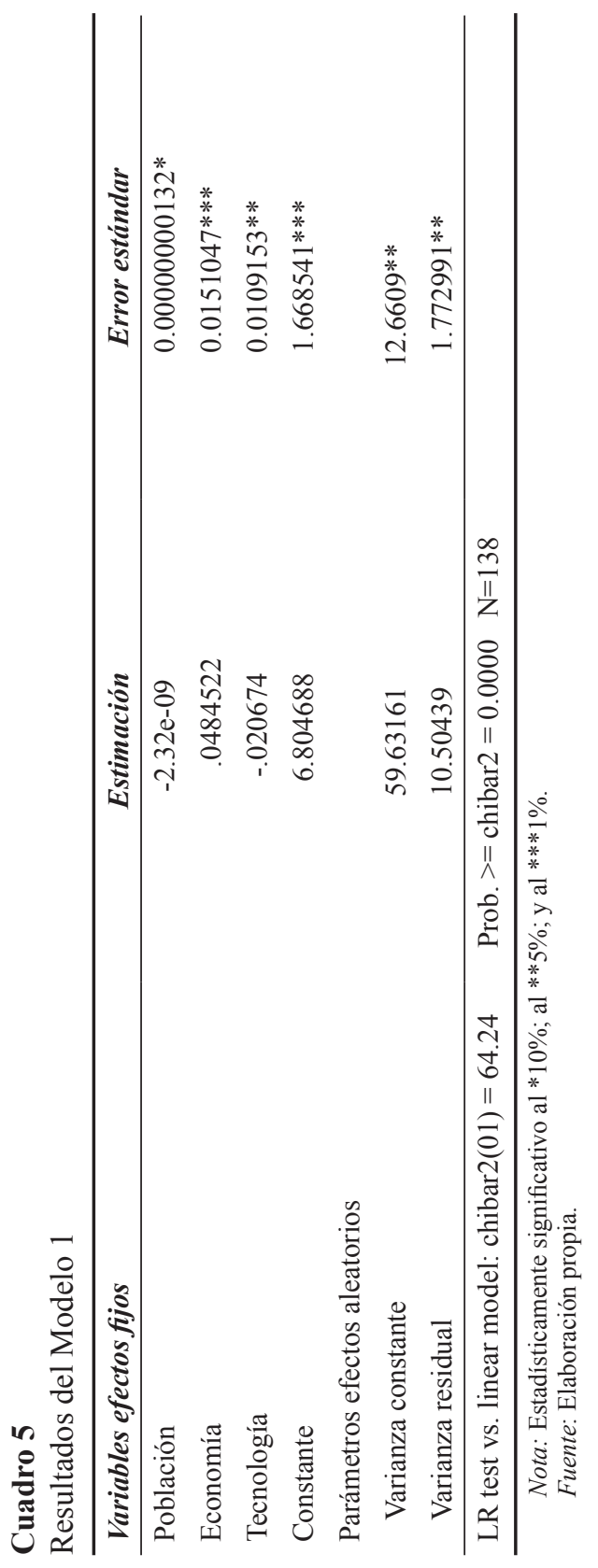




\section{Cuadro 6}

Resultados del Modelo 2

\begin{tabular}{lcl}
\hline Variables efectos fijos & Estimación & Error estándar \\
\hline Población & $-2.24 \mathrm{E}-09$ & $0.00000000127^{*}$ \\
Economía & 0.0497614 & $0.0149153^{* * *}$ \\
Tecnología & -0.012532 & 0.0138934 \\
Constante & 5.814656 & $1.457467^{* * *}$ \\
Parámetros efectos aleatorios & & \\
$\quad$ Varianza tecnología & 0.0023706 & $0.0012105^{* *}$ \\
Varianza de la constante & 28.84556 & $12.65395^{* *}$ \\
$\quad$ Varianza residual & 9.627042 & $1.788097^{* *}$ \\
\hline LR test vs. linear model: chi2 $(2)=69.17 \quad$ Prob. > chi2 $=0.0000 \quad \mathrm{~N}=138$ \\
\hline
\end{tabular}

Nota: Estadísticamente significativo al $* 10 \%$; al $* * 5 \%$; y al $* * * 1 \%$.

Fuente: Elaboración propia.

\section{Cuadro 7}

Resultados del Modelo 3

\begin{tabular}{lcc}
\hline \multirow{2}{*}{ Variables } & \multicolumn{2}{c}{ Modelo completo } \\
\cline { 2 - 3 } Población & Estimación & Error estándar \\
Economía & $-2.32 \mathrm{E}-09$ & $0.00000000134 *$ \\
Tecnología & 0.0426532 & $-2.08 \mathrm{E}-02 * * *$ \\
Constante & -0.020811 & $0.0109877 * *$ \\
Parámetros efectos aleatorios & 6.599857 & $6.599857 * * *$ \\
$\quad$ Varianza IDI & $5.91 \mathrm{E}-21$ & $3.49 \mathrm{E}-20 * *$ \\
$\quad$ Varianza desempleo país & 0.2433089 & $0.1341318 * *$ \\
$\quad$ Varianza industria & 0.0045324 & $0.0156749 * *$ \\
$\quad$ Varianza de la constante & 19.56297 & $19.88209 * *$ \\
$\quad$ Varianza residual & 10.89359 & $1.899799 * *$ \\
\hline LR test vs. linear model: chi2(4) $=72.40$ & Prob. $>$ chi2 $=0.0000$ \\
\hline
\end{tabular}

Nota: Estadísticamente significativo al $* 10 \%$; al $* * 5 \%$; y al $* * * 1 \%$.

Fuente: Elaboración propia. 
Cuando se incluyen variables explicativas de nivel país, se observa que Tecnología conserva su efecto negativo y estadísticamente significativo al $5 \%$. A su vez, la variable Población posee un efecto sobre el desempleo significativo al $10 \%$ (al igual que en los modelos anteriormente estimados) y de signo negativo, tal como predice la literatura.

Tanto la variable IDI como las variables Desempleo país e Industria tienen efectos aleatorios sobre la tasa de desempleo de la ciudad. Los países difieren en cuanto a las políticas de desarrollo en TIC como en las políticas de empleo nacional y de industrialización, con implicancias en las tasas de desempleo urbano.

Por último, los datos del Cuadro 8 indican que el último modelo que incluye efectos aleatorios en las variables explicativas de nivel país (Tasa de desempleo país, Nivel de desarrollo en TIC e Industria) es el que explica un mayor porcentaje de la varianza total respecto del modelo nulo (69.28\%). También es el modelo que explica un mayor porcentaje de la varianza determinada por las variables sobre el modelo nulo a nivel países. Por el contrario, el Modelo 2 es el que explica un mayor porcentaje de la varianza sobre el modelo nulo a nivel ciudades; es decir, los efectos aleatorios como consecuencia de las diferencias en tecnología de las ciudades. Por lo tanto, el Modelo 3 es el seleccionado, modelo con efectos aleatorios en las variables TIC de nivel país. De esta manera, con el Modelo 3 se corrobora la importancia de las variables Desempleo, Tecnología e Industria de nivel país para la explicación del desempleo en las ciudades.

\section{Cuadro 8}

Comparación de modelos

\begin{tabular}{lrrrr}
\hline Varianza & $\begin{array}{c}\text { Modelo } \\
\text { nulo }\end{array}$ & Modelo 1 & Modelo 2 & Modelo 3 \\
\hline Varianza entre países & 63.68732 & 59.63161 & 28.84556 & 19.56297 \\
Varianza entre ciudades & 11.72008 & 10.50439 & 9.627042 & 10.89359 \\
Varianza total & 75.4074 & 70.136 & 38.4726 & 30.45656 \\
$\begin{array}{l}\text { Porcentaje de varianza sobre } \\
\text { el modelo nulo }\end{array}$ & & & & \\
$\quad$ Nivel países & 7.100487 & 6.368159 & 54.70753 & $\mathbf{6 9 . 2 8 2 7 9}$ \\
$\quad$ Nivel ciudades & 7.954895 & 10.37271 & $\mathbf{1 7 . 8 5 8 5 6}$ & 7.051914 \\
$\quad$ Total & 7.232049 & 6.990561 & 48.98034 & $\mathbf{5 9 . 6 1 0 6 5}$ \\
\hline
\end{tabular}




\section{Discusión de resultados y conclusiones}

La contribución principal de este trabajo consiste en estudiar la influencia de las Tecnologías de la Información y de la Comunicación (TIC) sobre la tasa de desempleo de las ciudades. A través de la estimación de modelos de tipo multinivel, con datos a nivel países y ciudades para el año 2016, se plantea el rol que cumplen ambos niveles de análisis para explicar el efecto de las tecnologías en la tasa de desempleo de las ciudades.

Los resultados obtenidos permiten observar que la tecnología a nivel de las ciudades es una variable estadísticamente significativa para explicar las mayores tasas de desempleo urbano, y que la tecnología a nivel países también influye sobre dichos niveles de desempleo. Es decir, ambos niveles de desarrollo tecnológico se complementan para determinar los niveles de desempleo registrados en las ciudades. Los resultados obtenidos confirman la visión de Clayton, Macdonald y Wilcock (2010) y Clayton y Macdonald (2013) de que las nuevas tecnologías son una de las causas de los problemas sociales, incluidos el desempleo y los bajos ingresos. Asimismo, siguen los resultados hallados por Acemoglu y Restrepo (2017), quienes analizan el efecto de los robots sobre el desempleo.

De acuerdo con los resultados del modelo estimado, los países difieren en su accionar respecto a las diferencias de origen en los niveles de desarrollo en TIC (IDI). Sin embargo, no es posible confirmar que puedan elaborar estrategias o líneas de acción en torno a las diferencias de origen en los niveles tecnológicos de las ciudades. Esto plantea la necesidad de reforzar el rol de los actores y gobiernos locales para enfrentar los desafíos que las nuevas tecnologías introducen en el mercado laboral. En este sentido, el trabajo confirma las ideas de Porter (1990) y Pearson (2006) respecto a la importancia del rol de las ciudades y las regiones para mejorar el bienestar; y de Tolga Akcura y Burcu Avci (2014) en cuanto a la responsabilidad de la gobernanza urbana para crear trabajos. Por otro lado, las variables de control, tanto a nivel ciudad (indicadores económicos y demográficos) como país (tasa de desempleo), explican las tasas de desempleo en el ámbito urbano.

Las limitaciones del trabajo responden en parte a la disponibilidad de información a nivel ciudad. Es difícil tener acceso a datos sobre producto bruto a nivel urbano o porcentaje de trabajadores con estudios secundarios o universitarios completos que permitan introducir más directamente la economía y la educación urbanas como variables de control en el trabajo. De todos modos, esto no invalida los resultados obtenidos del modelo bajo el supuesto de una posible relación espuria entre la tecnología TIC y el desarrollo humano. Si se considera el Índice de Desarrollo Humano IDH como un indicador apropiado de desarro- 
llo a nivel países, debe recordarse que este índice compuesto introduce varias dimensiones, tales como salud, educación e ingreso familiar. Estas últimas ya se encuentran contempladas en el modelo a través de las variables Economía e IDI, el cual incluye el nivel educativo de la población en el ámbito países.

A su vez, si bien existe cierto consenso en que las TIC pueden fomentar el desarrollo (Qureshi, 2011), el acceso a las computadoras e internet, así como otros indicadores de naturaleza más técnica o de acceso, brindan una aproximación incompleta del desarrollo digital (Armenta et al., 2012; Morawczynski y Ngwenyama, 2007). En esta línea, Alderete (2016) encuentra que el acceso a las TIC tiene impacto en el desarrollo socioeconómico de los países en la medida que sea moderado por el efecto del uso y habilidades en las TIC. Este resultado se sostiene tanto para el grupo de países desarrollados como en desarrollo. Por lo tanto, no necesariamente mayor tecnología conduce a mayor desarrollo.

Por otro lado, sería interesante disponer de información sobre ciudades no metropolitanas, ya que, como se ha mencionado en la metodología, la mayoría de las urbes son ciudades capitales o metropolitanas. Asimismo, sería deseable replicar el trabajo con datos en el tiempo para poder explorar el efecto de los cambios en las TIC en la tasa de variación del empleo.

\section{Anexo}

Ciudades que conforman la muestra

\begin{tabular}{|c|c|c|c|}
\hline País & Ciudades & País & Ciudades \\
\hline $\begin{array}{l}\text { Alemania } \\
\text { Arabia Saudita } \\
\text { Argentina } \\
\text { Australia } \\
\text { Austria } \\
\text { Bélgica } \\
\text { Bolivia } \\
\text { Bosnia } \\
\text { y Herzegovina } \\
\text { Brasil }\end{array}$ & $\begin{array}{l}\text { Berlín, Munich, } \\
\text { Frankfurt, Hamburgo, } \\
\text { Sttutgart, Colonia } \\
\text { Jidda } \\
\text { Buenos Aires, } \\
\text { Córdoba, Rosario } \\
\text { Sídney, Melbourne } \\
\text { Viena, Linz } \\
\text { Bruselas, Amberes } \\
\text { La Paz, Santa Cruz } \\
\text { Sarajevo } \\
\text { Porto Alegre, } \\
\text { São Paulo, Curitiba, } \\
\text { Brasilia, Río de } \\
\text { Janeiro, Recife, } \\
\text { Fortaleza, Salvador, } \\
\text { Belo Horizonte }\end{array}$ & $\begin{array}{l}\text { Colombia } \\
\text { Costa Rica } \\
\text { Croacia } \\
\text { Dinamarca } \\
\text { Ecuador } \\
\text { Emiratos } \\
\text { Árabes } \\
\text { Eslovaquia }\end{array}$ & $\begin{array}{l}\text { Sofía } \\
\text { Vancouver, Toronto, } \\
\text { Ottawa, London, } \\
\text { Montreal } \\
\text { Santiago } \\
\text { Hong Kong, } \\
\text { Guangzhou, Beijing, } \\
\text { Shanghái, Shenzhen, } \\
\text { Chongqing, Wuhan, } \\
\text { Shenyang, Suzhou } \\
\text { Medellín, Bogotá, Cali } \\
\text { San José } \\
\text { Zagreb } \\
\text { Copenhague } \\
\text { Quito, Guayaquil } \\
\text { Dubái } \\
\text { Bratislava }\end{array}$ \\
\hline
\end{tabular}




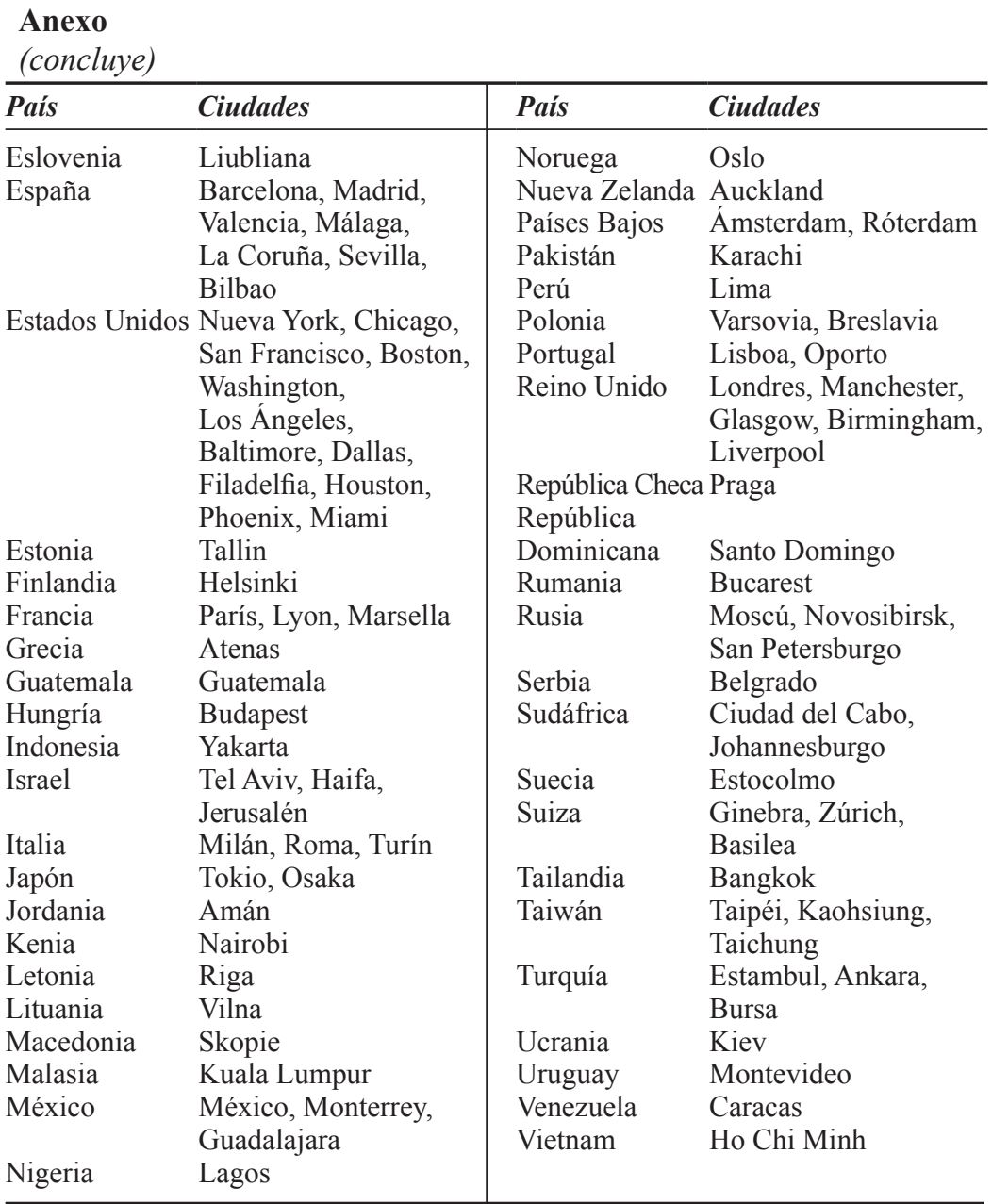




\section{Bibliografía}

Acemoglu, D. y Restrepo, P. (2017). Robots and jobs: Evidence from US labor markets. NBER Working Paper, 23285. Recuperado de http://www. nber.org/papers/w23285

Alderete, M. V. (2016). Examining the ICT access effect on socio-economic development: The moderating role of ICT use and skills. Information Technology for Development, 23(1), 42-58. Recuperado de https://doi. org/10.1080/02681102.2016.1238807

Alderete, M. V. y Gutiérrez, L. H. (2012). TIC y productividad en las industrias de servicios en Colombia. Lecturas de Economía, 77, 163-188. Recuperado de http://www.scielo.org.co/pdf/le/n77/n77a5.pdf

Armenta, A., Serrano, A., Cabrera, M. y Conte, R. (2012). The new digital divide: The confluence of broadband penetration, sustainable development, technology adoption and community participation. Information Technology for Development, 18(4), 345-353.

Audretsch, D. (1995). The innovation, unemployment and competiveness challenge in Germany. Londres: Centre for Economic Policy Research.

Autor, D. H. y Dorn, D. (2013). The growth of low-skill service jobs and the polarization of the U.S. labor market. American Economic Review, 103(5), 1553-1597. Recuperado de http://www.ddorn.net/papers/AutorDorn-LowSkillServices-Polarization.pdf

Barrón, A. (2013). Desempleo entre los jornaleros agrícolas, un fenómeno emergente. Problemas del Desarrollo. Revista Latinoamericana de Economía, 44(175), 55-79. Recuperado de http://www.redalyc.org/articulo. oa? id $=11828895004$

Belissent, J. y Giron, F. (2013). City service providers accelerate smart projects. Forrester Research Report. Cambridge, MA: Forrester Publication.

Bernal-Verdugo, L. E; Furceri, D. y Guillaume, D. (2012). Labor market flexibility and unemployment: New empirical evidence of static and dynamic effects. Comparative Economic Studies, 54(2), 251-273. Recuperado de https://www.imf.org/external/pubs/ft/wp/2012/wp1264.pdf

Brynjolfsson, E. y Mc Afee, A. (2015). The second machine age. Nueva York: W.W. Norton \& Company.

Castells, M. y Cardoso, G. (2005). The network society. From knowledge to policy. Washington, DC: Johns Hopkins Center for Transatlantic Relations.

Clayton, J. y Macdonald, S. (2013). The limits of technology. Information, Communication \& Society, 16(6), 945-966. Recuperado de https://www. tandfonline.com/doi/abs/10.1080/1369118X.2012.748817 
Clayton, J., Macdonald, S. J. y Wilcock, A. (2010). City of Sunderland Digital Inclusion Evaluation: Final report. Sunderland: University of Sunderland.

Crivelli, E., Furceri, D. y Toujas-Bernate, J. (2012). Can policies affect employment intensity of growth? A cross-country analysis. International Monetary Fund IMF Working Paper, 12/218. Recuperado de: https://ssrn.com/abstract=2169762.

Dameri, R. (2014), Smart city. How to create public and economic value with high technology in urban space. Nueva York: Springer.

De la Cruz, F. (2008). Modelos multinivel. Revista Peruana de Epidemiología, 12(3), 1-8. Recuperado de http://www.redalyc.org/articulo. oa? $\mathrm{id}=203120335002$

Diez Roux, A. (2002). A glossary for multilevel analysis. Journal of Epidemiology and Community Health, 56, 588-594. Recuperado de https:// jech.bmj.com/content/jech/56/8/588.full.pdf

Florida, R. y Mellander, C. (2012). The rise of skills: Human capital, the creative class and regional development. CESIS Electronic Working Paper Series, 266. Recuperado de https://static.sys.kth.se/itm/wp/cesis/ cesiswp266.pdf

Freeman, C., Clark, J. y Soete, L. (1983). Desempleo e innovación tecnológica. Un estudio de las ondas largas y el desarrollo económico. Sussex: University of Sussex.

Furuholt, B. y Kristiansen, S. (2007). A rural-urban digital divide? Regional aspects of internet use in Tanzania. The Electronic Journal of Information Systems in Developing Countries, 31(6), 1-15. Recuperado de https:// onlinelibrary.wiley.com/doi/abs/10.1002/j.1681-4835.2007.tb00215.x

García Sánchez, A. (1993). Cambio tecnológico y desempleo. Economía y Sociología del Trabajo, 19-20, 47-63.

García, B. y Sánchez, L. (2012). Trayectorias del desempleo urbano en México. Revista Latinoamericana de Población, 10(6), 5-29. Recuperado de http://www.alapop.org/alap/Revista/Articulos/relap10art1.pdf Gaviria, J. L. y Castro M. (2005). Modelos jerárquicos lineales. Madrid: La Muralla

Giles, J., Park, A. y Zhang, J. (2005). What is China's true unemployment rate?. China Economic Review, 16, 149-170. Recuperado de https:// www.sciencedirect.com/science/article/pii/S1043951X0400077X

Gomulka, S., Ostaszewski, A. y Davies, R. (1990). The innovation rate and Kalecki's theory of trend, unemployment and the business cycle. Economica, 57(228), 525-540. Recuperado de https://www.jstor.org/ stable/2554717?seq=1\#page_scan_tab_contents 
González Oliva, J. J., Karlsdotter, K., Martín, J. J. y Del Amo González, M. (2010). Factores que influyen en la creación de empleo para los asistentes a cursos de formación para el empleo en Andalucía. Investigaciones de Economía de la Educación, 5, 1223-1242. Recuperado de http://repec. economicsofeducation.com/2010zaragoza/05-61.pdf

Goos, M. y Manning, A. (2007). Lousy and lovely jobs: The rising polarization of work in Britain. The Review of Economics and Statistics, 89(1), 118-133. Recuperado de https://www.mitpressjournals.org/doi/ $\mathrm{abs} / 10.1162 /$ rest.89.1.118? journalCode $=$ rest

Hollands, R. (2008). Will the real smart city please stand up?. City, 12(3), 303-320. Recuperado de https://www.tandfonline.com/doi/full/10.108 $0 / 13604810802479126$ ?scroll $=$ top\&needAccess $=$ true

Hox, J. (2002). Multilevel analysis: Techniques and applications. New Jersey: Lawrence Erlbaurn Associates.

Hox, J. J. (1995). Applied multilevel analysis. Amsterdam: TT-Publikaties. Jaimovich, N. y Siu, E. (2012). The trend is the cycle: Job polarization and jobless recoveries (NBER Working Paper, 18334). National Bureau of Economic Research. Recuperado de http://www.nber.org/papers/w18334 Jolías, L. y Prince, A. (2016). Definiendo un modelo de smart cities para el contexto argentino. En N. Capellán (coord.), Ciudades inteligentes. El aporte de las TIC a la comunidad. Casos testigo y la visión del sector privado. Buenos Aires: Cámara de Informática y Comunicaciones de la República Argentina.

Katz, R. y Callorda, F. (2015). Impacto de arreglos institucionales en la digitalización y el desarrollo económico de América Latina. Proceedings of the 9th CPRLatam Conference, Cancún, 13 y 14 de julio. Recuperado de https://papers.ssrn.com/sol3/papers.cfm?abstract_id=2713990\#

Khattab, N. (2006). Ethnic and regional determinants of unemployment in the Israeli labour market: A multilevel model. Regional Studies, 40, 93105. Recuperado de https://www.tandfonline.com/doi/pdf/10.1080/003 43400500450083?needAccess $=$ true

Licht, G. y Moch, D. (1999). Innovation and information technology in services. Canadian Economic Review, 32(2), 363-383. Recuperado de $\mathrm{http} / / / \mathrm{www} . c s l s . c a /$ journals/sisspp/v32n2_06.pdf

Manpower Group (2017). The skills revolution. Digitization and why skills and talent matter. Recuperado de http://www.manpowergroup.co.uk/ the-word-on-work/skills-revolution/

Maroto-Sánchez, A. (2012). Productivity in the services sector. Conventional and current explanations. The Service Industries Journal, 32(5), 719-746. 
Recuperado de https://www.tandfonline.com/doi/full/10.1080/0264206 9.2010.531266? scroll=top\&needAccess $=$ true

McQuaid, R. W., Lindsay, C. y Greig, M. (2004). Reconnecting' the unemployed: Information and communication technology and services for jobseekers in rural areas. Information, Communication \& Society, 7(3), 364-388. Recuperado de https://www.tandfonline.com/doi/abs/10.1080/ 1369118042000284605

Michaels, G., Natraj, A. y Van Reenen, J. (2014). Has ICT polarized skill demand? Evidence from eleven countries over 25 years. The Review of Economics and Statistics, 96(1), 60-77. Recuperado de http://eprints. lse.ac.uk/46830/1/Michaels_Natraj_VanReenen_Has-ICT-polarizedskill-demand_2014.pdf

Morawczynski, O. y Ngwenyama, O. (2007). Unraveling the impacts of investments in ICTs, education and health on development: An analysis of archival data of five west African countries using regression splines. Electronic Journal on Information Systems in Developing Countries, 29(5), 1-15. Recuperado de https://onlinelibrary.wiley.com/doi/ pdf/10.1002/j.1681-4835.2007.tb00199.x

Mwesige, P. (2004). Cyber elites: A survey of internet café user in Uganda. Telematics and Informatics, 21(1), 83-101. Recuperado de https://www. sciencedirect.com/science/article/pii/S0736585303000248

Nath, H. K y Liu, L. (2017). Information and communications technology (ICT) and services trade. Information Economics and Policy, 41, 81-87. Recuperado de https://www.sciencedirect.com/science/article/pii/ S0167624516300646

Organización para la Cooperación y el Desarrollo Económicos (OCDE) (2003). PISA 2003. Manual de análisis de datos. Usuarios de SPSS. Madrid, España: OCDE.

Parkin, M., Esquivel, G. y Muñoz, M. (2007). Macroeconomía (7a. ed.). México: Pearson Educación.

Pearson, I. (2006). The role of future ICT in city development. Foresight, 8(3), 3-16. Recuperado de https://www.emeraldinsight.com/doi/ abs/10.1108/14636680610668036

Pianta, M. (2005). Innovation and employment. En J. Fagerberg, D. Mowery y R. Nelson (eds.), The Oxford handbook of innovation. Cap. 21 (pp. 568-598). Nueva York: Oxford University Press.

Pilat, D. (2001). Innovation and productivity in services: State of the art. En OECD (ed.), Innovation and productivity of services. París: OECD.

Porter, M. E. (1990). The competitive advantage of nations. Harvard Busi- 
ness Review, marzo-abril. Recuperado de https://hbr.org/1990/03/thecompetitive-advantage-of-nations

Qureshi, S. (2011). Globalization in development: Do information and communication technologies really matter? Information Technology for Development, 17(4), 249-252. Recuperado de https://www.tandfonline. com/doi/full/10.1080/02681102.2011.610142

Rao, S. (2005). Bridging digital divide: Efforts in India. Telematics and Informatics, 22(4), 361-375. Recuperado de https://www.sciencedirect. com/science/article/pii/S0736585305000195\#!

Rillo, G. (2015). Shocks and institutions, assessing the role of ICT on unemployment. Tesis de licenciatura, Universidad LUISS Guido Carli. Recuperado de https://tesi.luiss.it/15271/

Rubiales Pérez, M. (2016). Territorio y crisis: impacto territorial de la crisis económica en las regiones metropolitanas de Madrid y Barcelona. Scripta Nova. Revista Electrónica de Geografía y Ciencias Sociales, 20, 1-28. Recuperado de http://www.ub.edu/geocrit/sn/sn-549-7.pdf

Sapprasert, K. (2010). The impact of ICT on the growth of the service industries (Working Papers on Innovation Studies, 20070531). Centre for Technology, Innovation and Culture, University of Oslo. Recuperado de https://ideas.repec.org/p/tik/inowpp/20070531.html

Taylor, J. y Bradley, S. (1997). Unemployment in Europe: A comparative analysis of regional disparities in Germany, Italy and the UK. Kyklos, 50(2), 221-45. Recuperado de https://onlinelibrary.wiley.com/doi/ epdf/10.1111/1467-6435.00012

Thite, M. (2011). Smart cities: Implications of urban planning for human resource development. Human Resource Development International, 14(5), 623-631. Recuperado de https://www.tandfonline.com/doi/abs/ 10.1080/13678868.2011.618349

Tolga Akcura, M. y Burcu Avci, S. (2014). How to make global cities: Information communication technologies and macro level variables. Technological Forecasting \& Social Change, 89, 78-79. Recuperado de https://www.sciencedirect.com/science/article/pii/S0040162513002254

Trejo Nieto, A. B. (2017). Estructura regional y polarización económicopoblacional en el centro de México. Estudios regionales en economía, población y desarrollo. (Cuadernos de Trabajo de la UACJ, 38, 3-33). Recuperado de http://open-apps.uacj.mx/RePEc/cjz/ca41cj/Cuader nos\%20UACJ\%2038.pdf

United Nations (2006). World Economic and Social Survey 2006: Diverging growth and development. Disponible en https://www.un.org/development/ $\mathrm{desa} / \mathrm{dpad} / \mathrm{wp}$-content/uploads/sites/45/PDFs/WESS/2006wess.pdf 
Van Dijk, J. (2006). Digital divide research, achievements and shortcomings. Poetics, 34(4-5), 221-235. Recuperado de https://www.sciencedirect. com/science/article/pii/S0304422X06000167

Van Dijk, J. y Hacker, K. (2003). The digital divide as a complex dynamic phenomenon. The Information Society, 19(4), 315-326. Recuperado de https://ris.utwente.nl/ws/portalfiles/portal/6466527/Dijk03digital.pdf

Vicente, R. y López, A. (2006). Patterns of ICT diffusion across the European Union. Economics Letters, 93(1), 45-51. Recuperado de https://www. sciencedirect.com/science/article/pii/S016517650600125X

Wall, R. S. y van der Knaap, G. A. (2011). Sectoral differentiation and network structure within contemporary worldwide corporate networks. Economic Geography, 87(3), 267-308. Recuperado de https://onlinelibrary.wiley. com/doi/epdf/10.1111/j.1944-8287.2011.01122.x

Walravens, N. (2015). Mobile city applications for Brussels citizens: Smart city trends, challenges and a reality check. Telematics and Informatics, 32(2), 282-299. Recuperado de https://www.sciencedirect.com/science/ article/pii/S0736585314000641

$\mathrm{Wu}, \mathrm{L} . \mathrm{y}$ Brynjolfsson, E. (2013). The future of prediction: How google searches foreshadow housing prices and sales. SSRN Scholarly Paper. Rochester, NY: Social Science Research Network. Recuperado de: https://papers.ssrn.com/sol3/papers.cfm?abstract_id=2022293

\section{Sitios web}

Knoema: buscador de datos. En https://knoema.es/

\section{Acerca de la autora}

María Verónica Alderete es doctora en Economía por la Universidad Nacional del Sur (UNS), Argentina. Actualmente es investigadora adjunta en el Instituto de Investigaciones Económicas y Sociales del Sur (IIESS), Conicet-UNS. Es también profesora adjunta de Microeconomía en la UNS. Su investigación se centra en el área Sociedad de la Información, en relación con los temas de comercio electrónico, gobierno electrónico, modelos de adopción y productividad de las TIC, entre otros. Entre sus publicaciones recientes se encuentran:

Alderete, V. y Bacic, J. (2018). Local productive arrangements and local development in non-metropolitan municipalities of São Paulo, Brazil. Cuadernos de Gestión, 18(1), 103-123. 
Alderete, V. (2017). The age of prosumerism: Some micro-economic analysis. Journal of Theoretical and Applied Electronic Commerce Research, 12(3), 1-12.

Alderete, V. (2017). Mobile broadband: A key enabling technology for entrepreneurship? Journal of Small Business Management, 55(2), 254-269.

Fecha de recepción: 19 de mayo de 2017.

Fecha de aceptación: 16 de febrero de 2018. 